\title{
The use of elexacaftor/tezacaftor/ivacaftor in patients with cystic fibrosis post-liver transplant: a case series
}

\author{
Hunter Ragan ${ }^{1}$, Elizabeth Autry ${ }^{2}$, Taryn Bomersback ${ }^{3}$, Jennifer Hewlett ${ }^{4}$, Lauren \\ Kormelink $^{5}$, Julie Safirstein ${ }^{6}$, Laura Shanley ${ }^{4}$, and Lisa Lubsch ${ }^{7}$ \\ ${ }^{1}$ Goldfarb School of Nursing at Barnes-Jewish College \\ ${ }^{2}$ University of Kentucky Medical Center \\ ${ }^{3}$ Alberta Health Services \\ ${ }^{4}$ Children's Hospital of Philadelphia \\ ${ }^{5}$ University of Kentucky Health Care Pharmacy Services \\ ${ }^{6}$ Hospital of the University of Pennsylvania \\ ${ }^{7}$ Southern Illinois University Edwardsville - School of Pharmacy
}

June 21, 2021

\begin{abstract}
Introduction Cystic fibrosis (CF) related liver disease (CFLD) manifests as a wide spectrum of hepatobiliary disease and can progress to need liver transplantation. Elexacaftor/tezacaftor/ivacaftor (elx/tez/iva) is a cystic fibrosis transmembrane conductance regulator (CFTR) modulator which has superior efficacy compared to previously approved modulators. Use of elx/tez/iva, should be approached with caution in individuals with CFLD or following liver transplantation due to possible increases in LFTs and drug-drug interactions with several immunosuppressant medications. Objective The purpose of this case series was to explore if the use of elx/tez/iva is safe and tolerable in patients with CF post-liver transplantation. Methods A retrospective case series including patients prescribed elx/tez/iva following liver transplantation and an immunosuppressive regimen consisting of drug therapy metabolized by P-glycoprotein was completed. Results Ten patients at six CF centers with a median age of 22.1 years (range 14-43.4 years) and median time from transplant of 6.9 years (range 0.6-22 years) were included. Most patients $(8,80 \%)$ received a reduced or full dose of elx/tez/iva for a mean duration of 10.4 months (range 7-12 months). Fluctuations in LFTs occurred in all patients $(10,100 \%)$ and led to therapy discontinuation in two patients (20\%). Elx/tez/iva initiation resulted in elevations in tacrolimus trough concentration in 7 patients (70\%). Most patients who tolerated elx/tez/iva had symptomatic and quality of life improvement, increased body-mass-index, and maintained or improved lung function. Conclusion Initiation of elx/tez/iva in patients with $\mathrm{CF}$ who received a liver transplantation may be safe with clinical benefits.
\end{abstract}

\section{Title}

The use of elexacaftor/tezacaftor/ivacaftor in patients with cystic fibrosis post-liver transplant: a case series

\section{Authors}

Hunter Ragan, PharmD, MBA ${ }^{1}$

Elizabeth Autry, PharmD, BCPPS 2,3

Taryn Bomersback, BS Pharm, ACPR ${ }^{4}$

Jennifer Hewlett, PharmD ${ }^{5}$ 
Lauren Kormelink, PharmD, BCACP 2,3

Julie Safirstein, PharmD, BCPS ${ }^{6}$

Laura Shanley, PharmD ${ }^{5}$

Lisa Lubsch, PharmD, BCPPS, AE-C, FPPA ${ }^{1,7}$

\section{Goldfarb School of Nursing at Barnes-Jewish College, St. Louis, MO}

2 University of Kentucky HealthCare Department of Pharmacy Services, Lexington, KY

3 University of Kentucky College of Pharmacy, Lexington, KY

4 Alberta Health Services, Calgary, Alberta, Canada

5 Children's Hospital of Philadelphia, Philadelphia, PA

6 Hospital of the University of Pennsylvania, Philadelphia, PA

7 SSM Cardinal Glennon Children's Hospital, St. Louis, MO

\section{Keywords}

Elexacaftor/tezacaftor/ivacaftor (Trikafta), cystic fibrosis, cystic fibrosis related liver disease, liver transplant, biliary atresia

\section{Abbreviations}

AST: Aspartate transaminase

ALT: Alanine aminotransferase

ALP: Alkaline phosphatase

CF: Cystic fibrosis

CFLD: Cystic fibrosis related liver disease

CFTR: Cystic fibrosis transmembrane conductance regulator

$\mathrm{FEV}_{1}$ : Forced expiratory volume in one second

Elx/tez/iva: Elexacaftor/tezacaftor/ivacaftor

GGT: Gamma-glutamyl transpeptidase

LFT: Liver function test

ULN: Upper limit of normal

WNL: Within normal limits

$\mathrm{ppFEV}_{1}$ : Percentage of predicted forced expiratory volume in one second

\section{Abstract}

\section{Introduction}

Cystic fibrosis (CF) related liver disease (CFLD) manifests as a wide spectrum of hepatobiliary disease and can progress to need liver transplantation. Elexacaftor/tezacaftor/ivacaftor (elx/tez/iva) is a cystic fibrosis transmembrane conductance regulator (CFTR) modulator which has superior efficacy compared to previously approved modulators. Use of elx/tez/iva, should be approached with caution in individuals with CFLD or following liver transplantation due to possible increases in LFTs and drug-drug interactions with several immunosuppressant medications. 


\section{Objective}

The purpose of this case series was to explore if the use of elx/tez/iva is safe and tolerable in patients with $\mathrm{CF}$ post-liver transplantation.

\section{Methods}

A retrospective case series including patients prescribed elx/tez/iva following liver transplantation and an immunosuppressive regimen consisting of drug therapy metabolized by P-glycoprotein was completed.

\section{Results}

Ten patients at six CF centers with a median age of 22.1 years (range 14-43.4 years) and median time from transplant of 6.9 years (range 0.6-22 years) were included. Most patients $(8,80 \%)$ received a reduced or full dose of elx/tez/iva for a mean duration of 10.4 months (range 7-12 months). Fluctuations in LFTs occurred in all patients $(10,100 \%)$ and led to therapy discontinuation in two patients $(20 \%)$. Elx/tez/iva initiation resulted in elevations in tacrolimus trough concentration in 7 patients $(70 \%)$. Most patients who tolerated elx/tez/iva had symptomatic and quality of life improvement, increased body-mass-index, and maintained or improved lung function.

\section{Conclusion}

Initiation of elx/tez/iva in patients with $\mathrm{CF}$ who received a liver transplantation may be safe with clinical benefits.

\section{Background/Introduction}

Cystic fibrosis (CF) related liver disease (CFLD) manifests as a wide spectrum of hepatobiliary disease. The most common is focal biliary cirrhosis which results from biliary obstruction and progressive peri-portal fibrosis. The estimated prevalence of CFLD is approximately $10 \%$ with progression to liver failure requiring transplant in about $3 \%$ of individuals with CF. ${ }^{1,2,3}$

Approved in October 2019, elexacaftor/tezacaftor/ivacaftor (Trikafta, elx/tez/iva) is the newest CF transmembrane conductance regulator (CFTR) modulator for patients who are 12 years and older who have at least one F508del mutation or a mutation in the CFTR gene that is responsive based on in vitro data. ${ }^{4}$ $\mathrm{Elx} / \mathrm{tez} / \mathrm{iva}$ is superior to other modulator therapies with the clinical benefits of profound improvements in the percentage of predicted forced expiratory volume in one second $\left(\mathrm{ppFEV}_{1}\right)$, normalized sweat chloride concentration (which is a surrogate for CFTR function), better respiratory-related quality of life, rapid weight gain and less pulmonary exacerbation. ${ }^{5,6}$

There is warning and precaution regarding elevated bilirubin and LFTs with elx/tez/iva and individuals with CFLD require more frequent monitoring. ${ }^{4} \mathrm{~A}$ dose reduction is required if the benefit exceed the risk for use in patients with moderate hepatic impairment and use should be avoided in severe impairment based on the Child-Pugh classifications. ${ }^{4}$ There are additional concerns of drug-drug interactions with P-glycoprotein (PGP) substrates as elx/tez/iva is an inhibitor and will lead to increased serum concentrations of PGP substrates. ${ }^{4}$ Several immunosuppressants commonly utilized in solid organ transplant are substrates of PGP and have a narrow therapeutic index.

Guidelines for CFLD are outdated and are early in the process of being revised. Currently, there are no recommendations provided for CFTR modulator use in this patient population. ${ }^{2,3}$ There are no published data regarding the use of elx/tez/iva in patients after liver transplant and limited data exists for ivacaftor and tezacaftor in this patient population. This case series describes the use of elx/tez/iva in patients with CF post-liver transplant.

\section{Case Summaries}

Case 1 
A 14-year-old male with CF (F508del/F508del) who required a liver transplant in February 2019 for CFLD. He has not had any issues with rejection or other post-transplant complications. There was one hospitalization for transaminitis, and the etiology was presumed to be a viral infection with resolution by a week without any interventions. He was started on elx/tez/iva at the end of November 2019, nine months post-transplant. One tablet of elx/tez/iva was initiated in the morning and increased to two tablets in the morning after three weeks. LFTs and bilirubin levels were scheduled bimonthly along with bimonthly tacrolimus troughs. LFTs and bilirubin were mildly elevated after initiation and stabilized after two months of therapy. The tacrolimus concentration slightly fluctuated after elx/tez/iva initiation from 4.2 to $6 \mathrm{ng} / \mathrm{mL}$ (goal: 4-7 ng/mL). No other adverse events were reported. Before elx/tez/iva, gastrostomy tube feedings were required and after one month of therapy, his body mass index (BMI) increased from the $12^{\text {th }}$ percentile to the $48^{\text {th }}$ percentile. His baseline $\mathrm{FEV}_{1}$ percent predicted ( $\mathrm{ppFEV}_{1}$ ) was $90 \%$ in early 2019 , trended downward to $58 \%$ before elx/tez/iva initiation, and increased to $82 \%$. The patient reports overall improvement in quality of life and can take on more independent tasks.

Case 2

An 18-year-old female with CF (F508del/W1282X) who required a liver transplant in April 2015 for CFLD. Her post-transplant course was complicated by recurrent ascites and alloimmune hepatitis, requiring an inferior vena cava stent placement and hepatic vein dilation in December 2016 and revision in April 2017. Prior to therapy initiation, the patient intermittently had lower extremity edema. She was started on elx/tez/iva at the end of December 2019, 4.7 years post-transplant, at a reduced dose of one tablet of elx/tez/iva in the morning. After 3.6 weeks of therapy, the dose was titrated up to two tablets in the morning. Laboratory monitoring, including LFTs, bilirubin and tacrolimus levels, was conducted one day after therapy initiation, weekly for the first month and every two weeks thereafter. LFTs and bilirubin fluctuated, but peaked at 4 times the upper limit of normal (ULN) based on the laboratory reference range after one month at the increased dose. Concurrently, the patient had worsening lower extremity edema and new onset ascites prompting evaluation by her transplant center. The patient underwent a ballooning of her existing stent and had two additional stents placed. Elx/tez/iva was felt to not be a contributing factor in the patient's transaminitis as LFT values decreased following her procedure. The tacrolimus concentration increased to $22.3 \mathrm{ng} / \mathrm{mL}$ at one week after elx/tez/iva initiation prompting a dose reduction in tacrolimus. Following her elx/tez/iva dose increase, tacrolimus levels were between 3.6 to $7.3 \mathrm{ng} / \mathrm{mL}$ (goal: 3 to 8 $\mathrm{ng} / \mathrm{mL}$ ). The patient is tolerating elx/tez/iva without any reported adverse events. After eight months of therapy, the patient reported improved quality of life with minimal respiratory symptoms at baseline. The patient has not required any oral or systemic antibiotic therapy for a pulmonary exacerbation since initiating elx/tez/iva. Additionally, pulmonary function testing showed an improvement in $\mathrm{ppFEV}_{1}$ from $61 \%$ to $83 \%$.

\section{Case 3}

A 31-year-old male with CF (F508del/F508del) status post living donor liver transplant April 2019 for CFLD with esophageal varices, ascites, and cirrhosis was interested in elx/tez/iva therapy. After discussing with his liver transplantation team, he was switched from full dose tez/iva to elx/tez/iva in early November 2019 , seven months after his transplantation at full dose. Baseline ppFEV 1 was $90 \%$ and baseline LFTs and total bilirubin were within normal limits (WNL) at baseline and were ordered for two weeks after initiation. Two days after initiating therapy, the patient presented to the emergency department with complaints of sudden onset abdominal pain with diffuse tenderness to palpation acute tenderness to palpation in the left upper quadrant with positive guarding. On presentation, LFTs and bilirubin were stable WNL. Full workup was performed with no remarkable imaging findings on abdominal imaging. At the time of presentation, he was also diagnosed with a CF pulmonary exacerbation and admitted for intravenous antibiotic therapy. With all other causes reasonably excluded, the patient's pain was classified as a side effect of elx/tez/iva. Upon discharge five days later to complete his antibiotic course at home, the patient's abdominal pain had resolved and LFTs and bilirubin remained WNL. Repeat LFTs and bilirubin at one-week post discharge remained WNL with plans to continue to monitor every two weeks. At approximately one month after therapy initiation, $\mathrm{ppFEV}_{1}$ was 95 , but AST rose to 6 times the ULN, ALT increased to 2 times the ULN, 
and total bilirubin remained WNL. Repeat labs were ordered for one week later at which point the patient's AST and ALT were 5.7 and 5 times the ULN, respectively with total bilirubin WNL. At this time, elx/tez/iva therapy was discontinued and patient underwent testing for hepatic graft rejection. Hepatic graft rejection was ruled out and pathological evidence of drug induced liver disease was identified. His transplant team ordered labs for every two weeks for close monitoring of hepatic markers, which gradually trended back to WNL over several months. In May 2020, the patient was re-challenged with elx/tez/iva at reduced dose of one triple combination pill in the morning and one iva tablet in the evening. Labs were ordered weekly to monitor. At one week post re-initiation, his ALT and AST rose to 3.4 and 5.7 times the upper limit of normal respectively with total bilirubin of $1.4 \mathrm{mg} / \mathrm{dL}$, so therapy was discontinued. Again, liver function tests (LFTs) trended to WNL within about three months. In September 2020, he restarted full dose tez/iva by the transplant clinic. To date, LFTs have remained WNL.

\section{Case 4}

A 35-year-old male with CF (F508del/F508del) status post dual bilateral lung and liver transplant in late September 2018. Due to extensive sinus disease, the patient expressed interested in trialing elx/tez/iva, and as a collaborative agreement between his transplant team and CF care team, he initiated full dose elx/tez/iva therapy. Per his transplant center, labs were ordered weekly including a tacrolimus concentration, hemogram, and comprehensive metabolic panel. Baseline LFTs were WNL. At one week of therapy, the patient's LFTs remained WNL, but his tacrolimus concentration was $25.6 \mathrm{ng} / \mathrm{mL}$ (goal: $8-10 \mathrm{ng} / \mathrm{mL}$ ). That same day, he reported symptoms of tacrolimus toxicity including severe gastrointestinal upset and acute kidney injury was noted on labs with a serum creatinine of 1.6 times his baseline. His transplant center discontinued $\mathrm{elx} / \mathrm{tez}$ /iva the next day when the labs resulted. No pulmonary function outcomes were measured given that he was only on modulator therapy for about one week. At this point, the patient has not been re-challenged with elx/tez/iva therapy, and does not express interest in re-challenge in the future.

\section{Case 5}

An 18-year-old female with CF (F508del/F508del), short bowel secondary to meconium ileus and resection, and history of liver transplant as an infant secondary to biliary atresia and failed Kasai procedure. This patient had no episodes of acute or chronic rejection since the transplant, nor any other post-transplant complications. LFTs were predominantly normal (occasional intermittent elevations), with intermittently low platelets. Liver biopsies showed mild inflammatory changes (plasma cell predominant) with significant fibrosis dating back to 2010. Ultrasound in 2019 demonstrated stable, mild inhomogeneous echotexture of the liver with normal hepatic Doppler. This patient was initiated on full dose elx/tez/iva in March 2020. One week after starting therapy, her tacrolimus concentration tripled from 5.0 to $15.1 \mathrm{ng} / \mathrm{mL}$. During this time, no medication changes had been made, her overall medical condition remained stable, and LFTs remained normal. No adverse outcomes occurred as a result of the elevated tacrolimus concentration. The tacrolimus dose was reduced by $50 \%$ to attain her goal trough of $5 \mathrm{ng} / \mathrm{mL}$. Weekly tacrolimus concentrations thereafter showed declining trough levels which allowed for subsequent increases in tacrolimus dose. Within 1 month of starting elx/tez/iva the patient had returned to the tacrolimus dose she was using prior to initiation of $\mathrm{elx} / \mathrm{tez}$ /iva. LFTs were normal for the first month of therapy, but then became mildly elevated with normal bilirubin. The elx/tez/iva was continued with close monitoring. Her LFTs remained persistently mildly elevated for 7 months. Platelets decreased during this time period and INR was elevated. She underwent liver biopsy with further imaging and consultation. Upon completion of these investigations, it was believed this patient had developed cirrhosis (Child Pugh A) of the graft and chronic active hepatitis. There was no evidence of drug induced liver injury and it was felt the changes were not related to elx/tez/iva. She continued treatment with elx/tez/iva and is being managed by the liver transplant team for the findings of rejection/alloimmune hepatitis with the addition of mycophenolate mofetil. Clinically, the patient responded well to elx/tez/iva with reduced oxygen and BiPAP requirements, improved exercise tolerance, reduced cough, improved appetite, increased energy, and the ability to taper off morphine previously required to manage dyspnea. Her ppFEV 1 improved from $27 \%$ to $35 \%$. Over 7 months of treatment her BMI percentile improved from $1.9 \%$ to $9.1 \%$. 


\section{Case 6:}

A 21-year-old male with CF (F508del/F508del/Y301C) status post liver transplant in 2009 for CFLD. He was initially managed with corticosteroids and tacrolimus and then transitioned to tacrolimus monotherapy with a goal trough of 3-5 ng/mL. Since transplant, all transplant related labs have been remained within acceptable ranges. He has not had any episodes of rejection. His baseline best ppFEV1 during 2019 was 77\%. In December 2019, he started full dose elx/tez/iva. Baseline LFTs, bilirubin, and a tacrolimus trough were obtained prior to the start of therapy and were WNL. LFTs were repeated one week and two weeks after starting therapy and remained WNL. His tacrolimus concentration after one week was $3.3 \mathrm{ng} / \mathrm{mL}$ (goal: $3-4$ $\mathrm{ng} / \mathrm{mL}$ ) and was not repeated after two weeks due to stability. Labs were then spaced to three months after the start of therapy and again remained stable WNL including a tacrolimus of $3.6 \mathrm{ng} / \mathrm{mL}$. During therapy, he experienced a change in stool patterns including constipation and loose stools; however, this was difficult to differentiate from baseline stool issues. He also noted sometimes feeling more fatigued than previous in the evenings. Overall, since starting therapy his baseline cough diminished and respiratory symptoms improved though without marked change to $\mathrm{ppFE}_{1}$ which remained at $78 \%$.

Case 7

A 19-year-old female with CF (F508del and W1282X) status post liver transplant in October 2018 for CFLD. She was initially managed with corticosteroids and tacrolimus and then transitioned to tacrolimus monotherapy with a goal trough of 6-8 ng/mL. Since transplant, all transplant related labs have been remained within acceptable ranges. She has not had any episodes of rejection. Her baseline ppFEV 1 in 2019 was $73 \%$. She was initiated on full dose elx/tez/iva at the end of January 2020. Baseline LFTs and bilirubin were WNL and the tacrolimus trough was $7.9 \mathrm{ng} / \mathrm{mL}$. LFTs were repeated 1 week after starting therapy and were WNL. The repeat tacrolimus concertation was $8.8 \mathrm{ng} / \mathrm{mL}$ and as it was drawn one hour late which prompted a tacrolimus dosage reduction. No other therapies other than elx/tez/iva were started which could have impacted tacrolimus trough concentrations and she was noted to have no changes in diet or notable illnesses. Additionally, she also had an acute increase in total bilirubin to $1.5 \mathrm{mg} / \mathrm{dL}$ which was primarily unconjugated bilirubin of $1.4 \mathrm{mg} / \mathrm{dL}$. Repeat labs 1 week later remained WNL including a tacrolimus level of $7.7 \mathrm{ng} / \mathrm{mL}$. Her total bilirubin continued to be elevated with a level of $1.1 \mathrm{mg} / \mathrm{dL}$ which remained primarily unconjugated bilirubin of $1 \mathrm{mg} / \mathrm{dL}$. Labs were repeated 1 month after starting elx/tez/iva and all were stable and WNL except for a total bilirubin of $1.3 \mathrm{mg} / \mathrm{dL}$ which again was primarily unconjugated. After two months of elx/tez/iva, her labs remained stable and monitoring was spaced to every three months. No other adverse events have been reported. Her ppFEV ${ }_{1}$ increased to $100 \%$ after two weeks of therapy.

Case 8

A 21-year-old male with CF (F508del/F508del) status post liver transplant in 2012 for CFLD, complicated by post-transplant lymphoproliferative disorder (PTLD) diagnosed one-year post-transplant, which was successfully treated with rituximab. He has not had any issues with rejection since this time. His immunosuppression regimen includes sirolimus and prednisone. The patient was previously treated with lumacaftor/ivacaftor (lum/iva) and was transitioned to elx/tez/iva at the end of November 2019. Due to a stable hepatic panel on standard dosing of lum/iva, he was initiated on full-dose of elx/tez/iva. LFTs, bilirubin and sirolimus concentrations were scheduled for weekly monitoring after initiation of elx/tez/iva, however the patient was noncompliant with ordered frequency of blood draws, and initially, communication regarding aberrant labs was not successfully relayed between the liver transplant and CF providers. Therefore, due to unfamiliarity with this new CFTR modulator, when the patient's liver enzymes began to rise, the liver transplant team grew concerned for rejection. The patient was sent for liver biopsy on December 2019, which came back normal. A month later, the CF team was notified of significant elevations of up above 5 times the ULN in AST and ALT. Based on these elevations and concern for drug-induced hepatic impairment, elx/tez/iva dose was reduced to 2 tablets daily. After dose reduction, LFTs significantly improved. The patient's sirolimus concentration also increased significantly after the switch from lum/iva to elx/tez/iva, from $12.0 \mathrm{ng} / \mathrm{mL}$ (goal: $3-20 \mathrm{ng} / \mathrm{mL}$ ) to $24.1 \mathrm{ng} / \mathrm{mL}$ about 2 weeks later. Initially no change was made to sirolimus dose, despite discontinuation of the inducer lum/iva, which is likely what caused this jump in 
sirolimus concentration. The patient's sirolimus dose was incrementally reduced and returning to his dosing before lum/iva initiation, with sirolimus concentration returning to normal range. The patient's baseline $\mathrm{ppFEV}_{1}$ was $63 \%$, and after 3 months of therapy with elx/tez/iva, increased to $71 \%$, with corresponding improvement in symptoms and quality of life.

\section{Case 9}

A 22-year old female with a history of CF (F508del/Q493X), short gut syndrome due to bowel resection as an infant, status post liver transplantation in 1998 (liver failure was likely secondary to TPN), and autism spectrum disorder. Her immunosuppression regimen is tacrolimus monotherapy. She was started on voriconazole late September 2019 for allergic bronchopulmonary aspergillosis (ABPA), but therapy was discontinued due to LFT elevations and plan to initiate elx/tez/iva. Because of baseline LFT elevations and history of liver transplant, she was initiated on elx/tez/iva at a reduced dose of 2 tablets daily in November 2019. A week later, the CF team was notified by patient's mother that the patient had an unexpected seizure and was evaluated and treated at an outside hospital's emergency department (ED). Notably, the patient has no history of seizures, but does have a significant neuropsychiatric history of autism and developmental delay. Her workup, including electroencephalogram (EEG) and computed tomography (CT) of the head were normal. After this incident, elx/tez/iva was held for 1 week, and restarted at same dose in early December, with no further issues reported. After initiation of elx/tez/iva, LFTs were stable. The patient's tacrolimus concentration remained stable throughout treatment with elx/tez/iva without the need for dose changes. The patient's baseline ppFEV $\mathrm{F}_{1}$ was $67 \%$, and after 3 months of therapy with elx/tez/iva, remained stable at $68 \%$, however the patient experienced improvement in respiratory symptoms and improved quality of life.

\section{Case 10}

A 42-year-old male with history of CF (F508del/1716+1G- $>$ A) status post a combined liver/kidney transplant in 2003. His immunosuppression regimen includes tacrolimus and prednisone. He was initiated on full dose elx/tez/iva in early December 2019, with recommended weekly blood work to monitor hepatic panel and tacrolimus troughs. However, the patient was initially non-compliant with recommended blood work. Prior to initiation of elx/tez/iva, the patient's liver function was within normal limits, except for a slightly elevated AST of $46 \mathrm{IU} / \mathrm{L}$. LFTs and a tacrolimus concentration were finally collected in late March 2020, which revealed elevated level of $10.0 \mathrm{ng} / \mathrm{mL}$ (goal: $4-6 \mathrm{ng} / \mathrm{mL}$ ), elevated serum creatinine, and a stable hepatic panel. The tacrolimus dose was subsequently reduced by $50 \%$ and repeat a concertation was within goal range at $4.7 \mathrm{ng} / \mathrm{mL}$ and serum creatinine returned to baseline. The patient has not complained of any side effects since initiation of elx/tez/iva. The patient's baseline ppFEV 1 was $88 \%$, and after 3 months of therapy with elx/tez/iva, increased to $96 \%$, with corresponding improvement in respiratory symptoms and quality of life.

\section{Discussion}

Elexacaftor/tezacaftor/ivacaftor, the newest $C F T R$ modulator, is efficacious as demonstrated by improved $\operatorname{ppFEV}_{1}$, decreased respiratory symptoms and acute pulmonary exacerbation rates in patients with CF. However, use has not been well established in those with liver disease requiring transplantation. This case series describes the use of elx/tez/iva in ten individuals with CF post-liver transplantation, across six CF centers. This report found that introducing elx/tez/iva, in patients post-liver transplant, regardless of indication for transplant, was safe and showed a clinical benefit.

Elz/tez/iva was well tolerated by patients and the most common adverse events reported were elevations in LFTs. Trends in LFT elevations were mild and stabilized after a couple weeks of therapy. One patient discontinued therapy due to transaminitis, with a documented AST and ALT of 6 times the ULN. Other possible safety concerns were fatigue, stool frequency changes, and seizure.

In the presence of a known PGP drug interaction, this case series highlighted that with close monitoring of the immunosuppressive regimen, elx/tez/iva can be introduced. The most common starting dose was $200 \mathrm{mg} / 100$ 
$\mathrm{mg} / 150 \mathrm{mg}$ of elx/tez/iva, respectively. For those initiated at a reduced dose, dose escalation was performed based on LFT monitoring. For patients on tacrolimus, trough concentrations did fluctuate leading to dose adjustment in five $(55.6 \%)$ patients. Two patients reported tacrolimus toxicity with one patient discontinuing $\mathrm{elx} /$ tez/iva. This patient was started on full dose elx/tez/iva, which may suggest that initiation at lower doses with a slow titration may reduce the degree of fluctuation with trough concentrations and toxicity. Experience from these patients suggest that tacrolimus toxicity may be avoided with close monitoring early in elx/tez/iva therapy initiation. There was one patient on sirolimus that required a dose decrease, but this was most likely due to discontinuation of the CYP450 inducer, lum/iva. Refer to Table 2 for complete information on immunosuppression regimens.

Although use of this medication was only observed short-term, there was noticeable clinical improvement measured; for specific information refer to Table 3. Overall, this case series suggests that those with CFLD may experience clinical benefit with minimal adverse events.

\section{Conclusion}

Patients with CF who have received a liver transplant may be initiated on elx/tez/iva with demonstrated safety and clinical efficacy. Frequent LFT monitoring is warranted and can be used to guide elx/tez/iva dose titrations. Early and close therapeutic drug monitoring of immunosuppression is important to ensure safety and efficacy of the regimen. Additional research is necessary to determine long term safety and efficacy of elx/tez/iva in patients with CF status-post liver transplantation.

\section{References}

1. Cystic Fibrosis Foundation Patient Registry. Annual Data Report. Bethesda, Maryland; 2019.

2. Sokol RJ, Durie PR. Recommendations for management of liver and biliary tract disease in cystic fibrosis. Cystic Fibrosis Foundation Hepatobiliary Disease Consensus Group. J Pediatr Gastroenterol Nutr 1999;28 Suppl 1:S1-13.

3. Debray, Dominque, Kelly D, Houwen R, Strandvik B, Colombo C. Best practice guidance for the diagnosis and management of cystic fibrosis-associated liver disease. J Cyst Fibros 2011;10 Suppl 2:S29-36.

4. Trikafta (elexacaftor/tezacaftor/ivacaftor) [Package Insert]. Boston, MA: Vertex Pharmaceuticals Inc; 2020.

5. Middleton PG, Mall MA, Dřevínek P, Lands LC, McKone EF, Polineni D, Ramsey BW, Taylor-Cousar JL, Tullis E, Vermeulen F, Marigowda G, McKee CM, Moskowitz SM, Nair N, Savage J, Simard C, Tian S, Waltz D, Xuan F, Rowe SM, Jain R; VX17-445-102 Study Group. Elexacaftor-tezacaftor-ivacaftor for cystic fibrosis with a single Phe508del allele. N Engl J Med. 2019;381(19):1809-1819.

6. Heijerman HGM, McKone EF, Downey DG, Van Braeckel E, Rowe SM, Tullis E, Mall MA, Welter JJ, Ramsey BW, McKee CM, Marigowda G, Moskowitz SM, Waltz D, Sosnay PR, Simard C, Ahluwalia N, Xuan F, Zhang Y, Taylor-Cousar JL, McCoy KS; VX17-445-103 Trial Group. Efficacy and safety of the elexacaftor plus tezacaftor plus ivacaftor combination regimen in people with cystic fibrosis homozygous for the F508del mutation: a double-blind, randomised, phase 3 trial. Lancet. 2019;394(10212):1940-1948.

Table 1. Patient Demographics

\begin{tabular}{|c|c|c|c|c|c|c|}
\hline Case & Age (years) & Years Post- Transplant & Initial Dose & Final Dose & Duration (months) & Baseline AST / \\
\hline 1 & 14 & 1.75 & 1 tablet in $\mathrm{AM}$ & 2 tablets in $\mathrm{AM}$ & 12 & $24 / 32$ \\
\hline 2 & 18.5 & 5 & 1 tablet in $\mathrm{AM}$ & 2 tablets in $\mathrm{AM}$ & 7 & $70 / 74$ \\
\hline 3 & 31 & 0.6 & full dose & discontinued & 1 & $16 / 14$ \\
\hline 4 & 35 & 1.4 & full dose & discontinued & 0.25 & $12 / 14$ \\
\hline 5 & 18.3 & 17.3 & full dose & full dose & 8 & $29 / 12$ \\
\hline 6 & 21.8 & 11.8 & full dose & full dose & 11 & $66 / 25$ \\
\hline 7 & 19.5 & 2.1 & full dose & full dose & 10 & $30 / 44$ \\
\hline 8 & 22.3 & 8.75 & full dose & 2 tablets in $\mathrm{AM}$ & 12 & $50 / 67$ \\
\hline
\end{tabular}




\begin{tabular}{lllllll}
\hline Case & Age (years) & Years Post- Transplant & Initial Dose & Final Dose & Duration (months) & Baseline AST / A \\
\hline 9 & 22.9 & 22 & 2 tablets in AM & full dose & 12 & $16 / 26$ \\
10 & 43.4 & 17 & full dose & full dose & 11 & $46 / 36$ \\
\hline
\end{tabular}

Table 2: Immunosuppression

\begin{tabular}{llll}
\hline Case & Initial Immunosuppression regimen & Final Immunosuppression regimen & \% Change in immunosuppression \\
\hline 1 & Tacrolimus 2 mg BID & Tacrolimus 1.5 mg BID & 25\% dose reduction \\
2 & Tacrolimus 2.5 mg TID & Tacrolimus 1 mg TID & $60 \%$ dose reduction \\
3 & Tacrolimus 3 mg BID & Tacrolimus 4 mg BID & $33.3 \%$ dose increase \\
4 & Tacrolimus 7.5 mg BID & Tacrolimus 7.5 mg BID & No change* \\
& Sirolimus 10 mg daily & Sirolimus 10 mg daily & \\
5 & Tacrolimus 1.5 mg BID & Tacrolimus 1.5 mg BID & no change overall but 50\% reduction requ \\
6 & Tacrolimus 2 mg BID & Tacrolimus 2 mg BID & no change \\
7 & Tacrolimus 4.5 mg BID & Tacrolimus 4 mg BID & 11\% dose reduction \\
8 & Sirolimus 8 mg daily & Sirolimus 2 mg daily & $75 \%$ dose reduction** \\
9 & Tacrolimus 0.5 mg BID & Tacrolimus 0.5 mg BID & no change \\
10 & Tacrolimus 2 mg BID & Tacrolimus 1 mg BID & $50 \%$ dose reduction \\
\hline
\end{tabular}

*Elx/tez/iva dose decrease due to toxic tacrolimus concentration

** converted to elx/tez/iva from lum/iva

Table 3: Clinical Outcomes in Cases that Tolerated Elx/tez/iva

\begin{tabular}{lllllll}
\hline Case & Improved quality of life & Improved respiratory symptoms & Improved ppFEV $_{1}$ & Improved BMI $^{\text {LFTs WNL }}$ & In \\
\hline 1 & $\mathrm{X}$ & $\mathrm{X}$ & $\mathrm{X}$ & $\mathrm{X}$ & $\mathrm{X}$ & \\
2 & $\mathrm{X}$ & $\mathrm{X}$ & $\mathrm{X}$ & Stable & & \\
5 & $\mathrm{X}$ & $\mathrm{X}$ & $\mathrm{X}$ & $\mathrm{X}$ & & \\
6 & $\mathrm{X}$ & $\mathrm{X}$ & $\mathrm{X}$ & $\mathrm{X}$ & \\
7 & $\mathrm{X}$ & $\mathrm{X}$ & $\mathrm{X}$ & $\mathrm{X}$ & $\mathrm{X}$ & \\
8 & $\mathrm{X}$ & $\mathrm{X}$ & $\mathrm{X}$ & & \\
9 & $\mathrm{X}$ & $\mathrm{X}$ & $\mathrm{X}$ & $\mathrm{X}$ & $\mathrm{X}$ & $\mathrm{X}$ \\
10 & $\mathrm{X}$ & $\mathrm{X}$ & $\mathrm{X}$ & $\mathrm{X}$ & \\
Total & $100 \%$ & $100 \%$ & $75 \%$ & $87.5 \%$ & $62.5 \%$ & 25 \\
\hline
\end{tabular}

